\title{
Simple Models for Turbulent Self-Regulation in Galaxy Disks
}

\author{
Curtis Struck, and Daniel C. Smith \\ Dept. of Physics \& Astronomy, Iowa State University, Ames, IA 50011
}

Received — 


\begin{abstract}
Supernova explosions, and winds and energetic photon fluxes from young star clusters drive outflows and supersonic turbulence in the interstellar medium in galaxy disks, and provide broad spectrum heating which generates a wide range of thermal phases in the gas. Star formation, the source of the energy inputs, is itself regulated by heating and phase exchanges in the gas. However, thermohydrodynamic self-regulation cannot be a strictly local process in the interstellar gas, since galaxy disks also have a nearly universal structure on large scales.

We propose that turbulent heating, wave pressure and gas exchanges between different regions of disks play a dominant role in determining the preferred, quasi-equilibrium, self-similar states of gas disks on large-scales. In this paper we present simple families of analytic, thermohydrodynamic models for these global states, which include terms for turbulent pressure and Reynolds stresses. In these model disks star formation rates, phase balances, and hydrodynamic forces are all tightly coupled and balanced. The models have stratified radial flows, with the cold gas slowly flowing inward in the midplane of the disk, and with the warm/hot phases that surround the midplane flowing outward.

The models suggest a number of results that are in accord with observation, as well as some novel predictions, including the following. 1) The large-scale gas density and thermal phase distributions in galaxy disks can be explained as the result of turbulent heating and spatial couplings. 2) The turbulent pressures and stresses that drive radial outflows in the warm gas above and below the disk midplane also allow a reduced circular velocity there. This effect was observed by Swaters, Sancisi \& van der Hulst in NGC 891, a particularly turbulent edge-on disk. The models predict that the effect should be universal
\end{abstract}


in such disks. 3) Since dissipative processes generally depend on the square of the gas density, the heating and cooling balance in these models requires a star formation rate like that of the Schmidt Law. Conversely, they suggest that the Schmidt Law is the natural result of global thermohydrodynamical balance, and may not obtain in disks far from equilibrium.

Subject headings: Galaxies: Evolution — Galaxies: ISM — Galaxies: Structure — ISM: Kinematics and Dynamics — Turbulence 


\section{Introduction: Interstellar Gas in Galaxy Disks}

\subsection{Phase Structure and Turbulence}

The recognition that supernovae, massive star winds, and other impulsive energy inputs are important heat sources for the interstellar medium (ISM) in galaxies, and that they generate the warm and hot phases, was a turning point for the theory of the ISM in galaxies (e.g., McKee \& Ostriker 1977). Studies of giant expanding gas shells in our galaxy (e.g., Heiles 1984), and other nearby disk galaxies showed that the energies required to produce these structures are much greater than those produced by a single supernova or the winds of its stellar progenitor (see reviews of Brinks 1990, van der Hulst 1996). However, young clusters of hot stars and their multiple supernova do produce sufficient energy to make these supershells (e.g., Mac Low, McCray \& Norman 1989, Norman \& Ikeuchi 1989, Tenorio-Tagle, Rozyczka, \& Bodenheimer 1990, and Tomisaka 1992). With this realization the theory of superbubbles, breakout, chimneys and large-scale outflows developed quickly, and joined the older theory of galactic fountains (see Shapiro \& Field 1976, Bregman 1980, Cox 1981, Shapiro \& Benjamin 1991, and Schulman, Bregman, \& Roberts 1994) in contributing to our understanding of turbulent ISM heating and the disk-halo connection.

At about the same time, observational discoveries on the nature of several components of the ISM led to a improved understanding of its overall phase structure (see the reviews in the book of van der Hulst 1997). One important component is the warm neutral medium, WNM, or the Lockman layer in our galaxy. This component consists mostly of small HI clouds, and diffuse (cirrus) material distributed in a substantially thicker disk than the cold component (e.g., Dickey \& Lockman 1990, Malhotra 1995, and Haynes \& Broeils 1997). A second component is the warm ionized medium (WIM), or diffuse ionized medium (DIM), or the Reynolds layer in our galaxy, which is observed in $\mathrm{H} \alpha$ and other emission lines (e.g., Reynolds 1996, Rand 1997a). These two media are continuous and overlapping, though the 
WIM extends to greater scale heights above the disk than the WNM.

Both components have been studied in detail in our galaxy (see the review of Dickey \& Lockman 1990, and the recent HI study of Malhotra 1995), and are probably common constituents of late-type galaxies (see van der Hulst 1997). The line emission of the WIM makes it the easier component to study in other galaxies; it is observed in: 1) dwarf irregular galaxies (e.g., Hunter \& Gallagher 1997), 2) edge-on disk galaxies, especially the vigorously star-forming object NGC 891 (Howk \& Savage 1997, Rand 1996, 1997a,b, 1998, Swaters, Sancisi \& van der Hulst 1997), 3) large-scale outflows from nuclear starburst galaxies (e.g., Lehnert \& Heckman 1996), and 4) nearby disk galaxies at arbitrary inclinations (Wang, Heckman, \& Lehnert 1997). These studies also confirm the association of the various warm-to-hot components (henceforth, collectively WHM) with star formation regions, superbubbles, and large-scale outflows. This association, in turn, suggests connections with the still hotter ISM components observed in X-rays and radio continuum.

The question of how the extensive WHM is heated (and how the WIM is ionized) in galaxies that are not experiencing extensive starbursts is not entirely answered. Strong impulsive energy sources are quite localized, while the WHM is not, in either our galaxy or others (Rand 1996, Wang, et al. 1997). UV photofluxes can transfer energy over long distances, and cosmic rays and magnetic fields can also contribute to the pressure support. However, a variety of evidence indicates that other sources are needed to heat the WHM over most scales. The evidence includes the following: 1) fountain and superbubble models, with a good deal of mechanical energy injection, are very successful in accounting for the characteristics of the hot halo gas (Shapiro \& Field 1976, Bregman 1980, Li \& Ikeuchi 1992, McKee 1993), 2) an extra source of support beyond that associated with random motions of typical clouds is needed to support the Galactic HI (WNM) layer (Malhotra 1995), 3) the "disturbed" WIM in external galaxies seems to require additional energy source (Rand 
1997a,b, 1998, Wang et al. 1997), 4) observations of Faraday rotation in our galaxy (see summary in Minter \& Spangler 1996) demonstrate the existence of turbulence on small scales in the diffuse ionized gas, and models suggest that turbulent heating is important on those scales (Minter \& Spangler 1997, Minter \& Balser 1997). Turbulence, generated by the impulsive sources and propagated by (magneto)acoustic waves and mass flows may be the missing ingredient on the intermediate scales, as well as the large and small scales.

The idea that interstellar cloud structure is turbulent is decades old (see Larson 1979, Scalo 1987 and references therein). Hydrodynamic models of local regions of the ISM with heating and cooling sources included clearly illustrate the development of turbulence (e.g., Rosen \& Bregman 1995, Passot, Vazquez-Semadeni, \& Pouquet 1995). Chappell \& Scalo (1997) argue that clouds themselves are multifractal manifestations of the interstellar turbulence. Elmegreen (1997) agrees that interstellar clouds have a fractal structure, and

further proposes that the "holes and gaps" that make up the intercloud medium are the result of turbulent heating rather than "clearing" by supernova explosions. Norman and Ferrara (1996) calculate that the turbulent energy injection into the interstellar gas is characterized by a very broad band spectrum, and that the general turbulent pressure may exceed the thermal pressure by 1-2 orders of magnitude. Thus, there is increasing evidence that turbulence supplies a large part of the heating needed to maintain the continuous range of phases in the ISM, and much of the pressure support. This principle is the central assumption on which the models described below are based.

\subsection{Large-Scale Structure}

Heating and cooling processes in the interstellar gas generally have characteristic length scales of less than a kiloparsec or so. These local thermal processes co-exist with global regularities in the structure of gas-rich disks, like the nearly universal surface density 
profiles of the cold gas and stellar components. The profile forms of these components are often described as a negative exponential functions of radius in the disk, though the gas surface density is also well described as having a 1/r form (e.g., Struck-Marcell 1991). Kennicutt's (1989, 1990, 1998a) influential studies of star formation (henceforth SF) in galaxy disks showed that the neutral gas surface density varies with radius in such a way that it is always nearly equal to the radially dependent threshold density for gravitational instability. These results revived earlier suggestions that local gravitational instabilities are needed to assemble the massive clouds where star clusters are formed. The threshold surface density also varies as $1 / r$ if the circular velocity is a constant, independent of radius, as observed in many disks.

It is widely believed that the disk gas surface density profile is a result of initial conditions, and the disk formation process. The reader is referred to the papers of Steinmetz \& Müller (1995), Dalcanton, Spergel, \& Summers (1997), and Mo, Mao, \& White (1998) for recent discussions of disk formation and early evolution. However, as noted above, thermal and turbulent pressure forces, as well as gravity and centrifugal force, are important in the WHM, and this makes it less likely that initial profiles are "frozen out" in these media. The effects of turbulent pressure on the large-scale structure of the WHM, have not yet received much attention. In the context of the secular evolution of the cold gas, Struck-Marcell (1991) pointed out that a 1/r surface density profile was required to maintain the conditions of hydrostatic equilibrium in the disk with minimal transport by shear viscosity (see below). The same arguments apply to the WHM, and more strongly, because of its shorter sound-crossing timescale. This leads us to the hypothesis that in any disk where the heating by SF activity is sufficient to maintain and cycle a large fraction of the gas through the WHM, turbulent hydrodynamic forces will regulate the gas surface density to the $1 / r$ profile. Furthermore, the recent work of Martin (1998 and references therein) suggests that substantial cycling rates are quite plausible. This hypothesis provides 
a second pillar on which the models below are built.

Dopita (1985) described one of the first models of global self-regulation by star formation. His model was based on the assumption of equipartition between turbulent and thermal pressures. In the models derived below we will consider cases where the turbulent pressure exceeds the thermal pressure in the WHM phases, as suggested by Norman and Ferrara. This is analogous to the situation in the cold cloud ensemble, where cloud random velocities are supersonic. We will, however, assume pressure balance between different thermal phases.

Many mechanisms of self-regulation based on the intrinsic stability properties of local star-cloud systems in disks have been suggested. In particular, models with cloud buildup by collisional agglomeration and disruption by SF activity have been popular, since they often yield (Schmidt-type) SFRs with power-law dependences on local gas density (e.g., Scalo \& Struck-Marcell 1984, Struck-Marcell \& Scalo 1987, Dopita 1990, Dopita \& Ryder 1994). Paravano has extensively investigated how star formation can be regulated by the thermal conversion processes that operate between warm diffuse phases and the small cool clouds (1988, 1989, also see Franco \& Shore 1984, Diaz-Miller, Franco, \& Shore 1998, and Bertoldi \& McKee 1997 for related models). With reasonable approximations he finds that these processes also produce a Schmidt Law SFR.

Silk (1997, and references therein) has argued for a somewhat different star formation law, that includes a dependence on local shear, and has described self-regulation and a derivation of the Tully-Fisher relation in a model based on this law. Kennicutt (1998a,b) finds that the global properties of star formation in galaxies are consistent with both the Schmidt and Silk/Wyse phenomenological laws.

There are several difficulties with many of these approaches to self-regulation. The first is that most require some arbitrary phenomenological assumptions, i.e., about SF or 
cloud collision rates, equipartition, or constraints on the ambient pressure. The second is that most are local in the sense that they do not include the hydrodynamic couplings to adjacent regions. On the other hand, the so-called chemodynamical models are based on a large-scale hydrodynamical treatment. For example, Samland, Hensler, \& Theis (1997 and references therein) have recently presented two-dimensional hydrodynamical models with three stellar components and two discrete gaseous phases. Inevitably, there are many uncertain parametrizations in the couplings between the components, which limits the predictive power of the models. Moreover, they do not include the effects of turbulent stresses in the intercloud medium, which we feel are essential to an understanding of the large-scale structure. The primary role of these stresses distinguish the models below from most previous ones.

\section{Global Analytic Models with Multiphase Turbulence}

\subsection{Densities and Radial Velocities}

A good conceptual model of gas dynamics and star formation in galaxy disks requires a quasi-static solution of multiphase hydrodynamic equations that include the important thermohydrodynamic forces of self-regulation. Ideally, the model should be simple enough to allow a clear understanding of both the structure of individual disks and the universal relations between disks. As a step towards this goal we here introduce a model of a two-component ISM described by cylindrically symmetric hydrodynamic equations, with turbulent stress terms. The two components are a cold isothermal (cloud) phase and a mean WHM described by a locally adiabatic equation of state (see below). This two-phase model is a minimal description of the multiphase interstellar gas, but it is sufficiently complex to capture many interesting behaviors, as we will see below. We will describe a simple analytic version of the model here, but the model is readily generalizable to a continuous range of 
phases, albeit with much increased complexity in the equations. We plan to develop that generalization in a later paper. In this paper we do not consider the effects of magnetic forces or cosmic rays separately from the thermal or turbulent pressures.

As part of the definition of a "quasi-static" disk, we will assume that the mass exchanges between phases balance locally. Then the time-independent mass continuity equations are of the form,

$$
\Sigma_{i} v_{r i} r=\text { constant }
$$

where $\Sigma_{i}$ is the phase component surface density and $v_{r i}$ is the component radial velocity, and with $\mathrm{i}=\mathrm{c}, \mathrm{w}$ for the two phases. For convenience, we will frequently use the approximation that $\Sigma_{i}=\rho_{i} h_{i}$, where $\rho_{i}$ is the component mass density, and $h_{i}$ is the component scale height in the direction perpendicular to the disk.

We assume hydrostatic equilibrium in the vertical direction (i.e., perpendicular to the disk). When the disk self-gravity dominates the vertical potential gradient, then the solutions to the component hydrostatic equations give component scale heights that increase slowly with radius (as $r^{1 / 2}$, see Malhotra 1995 for a discussion of the application of this approximation to the Milky Way).

The zero radial flow solution, $v_{r c}=v_{r w}=0$, to equation (1) is often assumed. However, if $\Sigma_{i} \propto 1 / r$, then there is a more general family of solutions in which $v_{r i}(r)=$ constant. Especially interesting are the solutions with $v_{r c}=-\left(\Sigma_{w} / \Sigma_{c}\right) v_{r w}$, describing opposed radial flows in the two components, but with zero net radial mass transport. This allows the slow inflow of high density cold (or cooling) gas to replace gas heated into the warm phases by SF, which are on average flowing outward. The flow of individual mass elements might consist of little more than a circulation within a local fountain (like a transient convective 
cell), with the ensemble of fountains making up the global inflow/outflow solution. Figure 1 provides a schematic view.

Because the scale-height ratio $h_{c} / h_{w}$ is small, inflow dominates in the midplane of the disk, sandwiched between warm outflows above and below. The existence of two discrete radial velocities is a consequence of the assumption of two distinct phases, and the model can be generalized to a continuous range of phases as a function of distance from the midplane with continuously stratified radial velocities. The fact that the $1 / r$ profile is a good approximation to the observed cold gas distributions in large parts of many late-type galaxy disks (Struck-Marcell 1991) provides empirical support for the constant radial velocity inflow/outflow solutions as opposed to more complicated radius-dependent flows. But why should this profile be universal?

\subsection{Hydrodynamic Forces and the $1 / r$ Surface Density Profile}

Several decades of numerical studies suggest that when the cold gas density significantly exceeds the gravitational instability threshold in all or part of a disk, then the result is the rapid development of instabilities that generate readily observable clumps. (An example of the clumping instability in disk formation is presented in Noguchi 1998.) The massive clumps would generate strong SF and heating, which would regulate the cold gas to lower densities by the processes described above. Thus, we do not expect to find densities much in excess of the threshold, which scales as $1 / r$ in a flat rotation curve disk.

Hydrodynamical stability arguments also lead to a preference for the $1 / r$ surface density profile, as described by Struck-Marcell (1991). For example, if the mean random velocity of the cold cloud ensemble is a constant independent of radius (i.e., the ensemble is isothermal), then only the $1 / r$ profile yields a constant net pressure force between 
adjacent annuli in the disk. Any deviation from that profile generates a pressure gradient proportional to the deviation, which would allow the nonlinear amplification of disturbances. This is not consistent with a hydrodynamic steady state. As noted above, this argument is even stronger when applied to the WHM.

A qualitative, but very general argument, is based on the observation that there is no obvious characteristic scale (other than the scale of the rising rotation curve in the center) over the range extending from a few kiloparsec to tens of kiloparsecs in late-type disks. This suggests that the important forces within the disk all have essentially the same radial or distance scaling. If not, we would expect there to be observational signatures associated with changes in the dominant force. To make this point more definite, we write the radial momentum equation for the cold gas component as follows,

$$
v_{r c} \frac{\partial v_{r c}}{\partial r}=\left(\frac{-1}{\rho_{c}}\right) \frac{\partial P_{c}}{\partial r}-\left(\frac{G M_{o}}{r_{o}}\right) \frac{1}{r}+\frac{v_{\phi c}^{2}}{r}
$$

where $M_{o}, r_{o}$ are a gravitational scale mass and radius, respectively, and $v_{\phi c}$ is the azimuthal velocity in the cold component. The terms on the right-hand-side represent the pressure gradient, gravitational, the centrifugal accelerations.

In equation (2) the gravitational and centrifugal accelerations both have a $1 / r$ scaling. There is no power-law solution for $v_{r c}$ that yields the desired scaling for the advection term. However, when $v_{r c}$ is constant this term vanishes. Moreover, the isothermal equation of state also yields the correct scaling in the pressure gradient acceleration for any power-law density profile, though we generally expect this term to be negligible in the cold gas. Thus, the adopted density and velocity profiles make up the only self-similar family of steady solutions to equations (1) and (2).

The remaining momentum equations do not require any changes in the density and 
velocity scalings. The azimuthal momentum equations are described in the next section, the radial momentum equation for the WHM is,

$$
v_{r w} \frac{\partial v_{r w}}{\partial r}=\left(\frac{-1}{\rho_{w}}\right) \frac{\partial P_{w}}{\partial r}-\left(\frac{G M_{o}}{r_{o}}\right) \frac{1}{r}+\frac{v_{\phi w}^{2}}{r}+\alpha_{w} \frac{\Delta v_{T} \Delta v_{\phi}}{r}
$$

This equation is very similar to the previous one, and in both cases the left-hand-side is zero for the constant radial velocity models. One difference is that we expect the pressure gradient term, which contains both thermal and turbulent pressures, to be significant in this component, in contrast to the cold component. The additional last term derives from an effective turbulent shear viscosity in the azimuthal direction. This term must be included because the change in specific angular momentum due to "viscous" forces changes the effective centrifugal force from what we would expect in the absence of "viscosity". Thus, in the warm gas, the non-negligible pressure gradient, and the steady input of angular momentum allow gravity to be balanced with less centrifugal force, while maintaining a constant velocity outflow.

There is a corresponding rate of decrease of angular momentum in the cool gas, but assuming that the total mass and angular momentum of the cold gas are greater than the WHM, we have assumed that this term is negligible in equation (2). We will discuss the viscous shear terms further in the next section, but we note here that the term in equation (3) contains the quantity, $\Delta v_{\phi}$, which we define as $\max \left(v_{\phi c}-v_{\phi w}, \Delta v_{T}\right)$, and $\Delta v_{T}$ is the turbulent velocity dispersion in the WHM.

In this simple model we will assume that the viscous coefficients $\alpha_{i}$ in the two phases, the nearly circular azimuthal velocities $v_{\phi i}$ and the velocity dispersions are all constant. In the case of the velocities this assumption is in accord with observation, see van der Hulst (1997). The pressure gradient is due to the random motions of the cold gas elements, and not cloud internal pressures. 


\subsection{Viscosity and Pressure}

Many processes may contribute to the viscosity in the interstellar gas, including: viscous shear within the cloud ensemble, drag against the diffuse components, enhancements

of these by bars and spiral waves, turbulent and magnetohydrodynamic couplings, etc. At the same time, turbulent pressure and turbulent angular momentum transfers to the WHM can provide support against gravity, as well as driving the radial flow. As mentioned above, the support against gravity implies that the circular velocity of this medium can be less than that of the cold gas. If the two (or multiple) phases have substantially different rotation speeds, $\Delta v_{\phi}$, then we expect turbulent shear viscosity between vertical layers to be the dominant viscous term. Cold gas heated by SF will be mixed into the WHM via chimneys, fountains, and bubble shells, and will add angular momentum to the WHM. At the same time, cooling lumps or filaments of the WHM, with lower specific angular momentum than the cold gas, will rain onto the midplane, decreasing the angular momentum of the cool clouds that sweep them up. The net result will be somewhat like the friction between two thin disks forced together at different speeds.

With the assumption that this is the only significant viscous term we can write the steady azimuthal momentum equation as,

$$
\frac{v_{r i} v_{\phi i}}{r}=-\alpha_{i} \frac{\Delta v_{T} \Delta v_{\phi}}{r}
$$

where $\mathrm{i}=\mathrm{c}, \mathrm{w}$ as usual, and this equation is valid for both components, though the radial velocities have different signs. The right hand term can be understood as dimensionally similar to a Navier-Stokes kinematic viscosity term of the form - $\nu\left(\partial^{2} v_{\phi} / \partial z^{2}\right)$, with a viscosity coefficient of order $\nu \simeq \Delta v_{T} \lambda$. In this case, the "mean free path" $\lambda$ is of order the size of a typical chimney, fountain, or the turbulent zone around an SF region, which 
we assume to be roughly constant across the disk. The vertical second derivative we approximate as of order $\Delta v_{\phi} / h_{w}^{2}$, where as noted above, the WHM scale height $h_{w} \propto r^{1 / 2}$. The coefficient $\alpha_{i}$ is assumed to be of order unity. Although the viscous term in the above equation may look somewhat unusual, in fact, it is a variant of the usual $\alpha$-viscosity for supersonic turbulence, $\nu \propto l_{\text {turb }} v_{\text {turb }}$, with scales appropriate to this problem.

As noted above, we can understand the last term in equation (3) as a momentum source term equivalent to a reduction in the centrifugal acceleration due to azimuthal viscosity. We assume that the radial velocities are small, and so, radial viscous accelerations are negligible. The centrifugal reduction can be treated as an acceleration, which we estimate as,

$$
\frac{\partial v_{r i}}{\partial t} \simeq \frac{v_{r i} v_{\phi i}}{r}=-\alpha_{i} \frac{\Delta v_{T} \Delta v_{\phi}}{r}
$$

where the first equality is derived on the assumption that this acceleration must have a magnitude sufficient to reduce the azimuthal velocity to zero in a time $r / v_{r i}$. The second equality results from substituting the previous equation. It is an additional simplifying assumption that the coefficient on the right-hand-side of this equation equals that of the previous equation, though we expect them to be of the same order. This term is usually neglected in viscous disk studies because $v_{r i}$ is small, but it is important here. We will also see later that $v_{r c}<<v_{r w}$, so $\alpha_{c}<<\alpha_{w}$, which justifies neglecting the term in equation (2).

Because of the low densities and high temperatures that characterize most of the WHM, we generally expect radiative cooling timescales to be long, so in our simple model an adiabatic equation of state is more appropriate than an isothermal one. This raises a potential problem with the scaling of the pressure gradient acceleration. However, shock heating plays an important role in determining the temperature structure of the WHM, and the frequency and intensity of the shocks varies with radius. Since shocks generate 
entropy, this suggests that the mean specific entropy of the WHM varies with radius, and so, the adiabat of the warm gas must also vary with radius. In this simple model we write the equation of state for the warm gas as,

$$
P_{w, t h e r m}(r)=K(r) \rho_{w}^{\gamma}
$$

where $P_{w, \text { therm }}$ is the thermal pressure, $\gamma \simeq 5 / 3$, and the adiabatic constant $\mathrm{K}$ varies with radius. (In reality, it probably also varies with vertical height z.) In particular, we obtain the desired self-similar form for the pressure gradient term with a radially dependent adiabatic constant of the form, $K(r) \propto r$. If the warm gas is flowing outward, this increase in specific entropy would be the result of the cumulative effects of shocks (see Appendix A). It is interesting that while the warm phase is assumed to be locally adiabatic in this simple model, the variation of pressure (and observables that depend on thermal temperature) with radius is the same as a globally isothermal gas. (Note that in terms of surface density and surface pressure we have $\Pi=K_{S} \Sigma^{5 / 3}$, with $\Sigma \propto r^{-1}, \Pi \propto r^{-1}$, and $K_{S} \propto r^{2 / 3}$, and again, $\Pi \propto \Sigma$.

At this point, the structure of a minimal model is nearly completely defined by the empirically constrained $(1 / r)$ surface density profiles, the adopted equations of state for thermal pressures, the continuity equations, the radial and azimuthal momentum equations, and the condition of pressure balance between phases. Using the ideal gas law to relate (total) pressure $P_{w}$ to the temperature $T_{w}$, we have the following equation for the inter-phase pressure balance,

$$
\mathbf{R} \rho_{w} T_{w}(1+\beta)=\rho_{c}\left(\Delta v_{c}\right)^{2}
$$

where the $\mathbf{R}$ is the gas constant, $\beta(r)$ is a correction factor for the turbulent contribution to 
the total pressure in the WHM, and the right-hand-side represents the pressure in random cold cloud motions (probably also turbulence dominated). For simplicity and consistency, we assume that $\beta$ is a constant, and so, turbulent and thermal pressures scale with radius in the same way.

\section{Vertically-Dependent Circular Velocities and the Case of NGC 891}

In this model the turbulent pressure gradient can provide some support in the WHM against gravity, so the circular velocity of this medium can be smaller than that of the cold gas. Recently, Swaters, Sancisi, \& van der Hulst (1997) discovered that the rotation velocities of $\mathrm{HI}$ gas located in a plane parallel to, but above, the midplane of the edge-on galaxy NGC 891 are less than those in the midplane by $25-100 \mathrm{~km} / \mathrm{s}$. Over most of the disk the velocity difference was about $25 \mathrm{~km} / \mathrm{s}$. The highest values come from a couple

of points within a radius of about $6.0 \mathrm{kpc}$. In the case of these points, the gas above the midplane may have originated in an outflow from smaller radii, and thus, considerably down the solid body part of the rotation curve. If so, the large velocity offset may be due to conservation of the small angular momenta in this gas.

Swaters et al. provide several possible explanations for the general effect, including local outflows and the angular momentum effect just mentioned. They also mention the possibility of "asymmetric drift", though they are skeptical that velocity dispersions as large as suggested by the empirical formula for asymmetric drift in our galaxy are achievable. However, since galactic asymmetric drift is a phenomenon of collisionless stars on orbits with large epicycles, or quite flattened elliptical orbits, we do not believe it is relevant unless elements of the WHM can become isolated and collisionless for large parts of their orbits.

Instead, we believe that the general velocity offset could be the result of substantial 
turbulence in this vigorously star-forming object (see Rand 1996, 1997a,b, 1998, and Howk \& Savage 1997). We will provide a simple numerical example below of how the model can account for this phenomenon in this section. In fact, we view this phenomenon, together with the vertical radial velocity gradient, as essential predictions of the model. Thus, detailed rotation studies, like that of Swaters et al., in other nearby, edge-on disks with known "DIG" components like NGC 3079, NGC 4631, NGC 5775, NGC 4302 (Rand 1996 and references therein), and NGC 55 (Ferguson, Wyse, \& Gallagher 1996, Hoopes, Walterbos, Greenwalt 1996) should provide a strong test of the model.

\section{Scaling the Model}

We can now describe how to scale the model, and provide some concrete examples. For brevity, we will not consider boundary conditions here, but simply assume an infinite radius solution. We begin by initializing several observable quantities, and also the constant radial mass transfer rate $\left(\dot{M}=2 \pi r h_{i} \rho_{i} v_{r i}\right)$, which is not generally observed. However, this quantity is related to the global SFR, since SF activity is responsible for the viscous and pressure forces. Numerical simulations should eventually yield a relation between them. Martin's (1998) studies of dwarf galaxies found the intriguing result that "shells lift gas out of the disk at rates comparable to, or even greater than, the current galactic star formation rates." This finding lends much credibility to the idea that both radial mass transfer and phase exchanges occur in late-type disks at interesting rates.

The remaining quantities we initialize are $\rho_{c}\left(r_{o}\right), \Delta v_{c}\left(r_{o}\right), \Delta v_{T}\left(r_{o}\right)$, and $\beta$. In principle, these are all evaluated at a particular radius $r_{o}$. In fact, the last three are constrained to be constants, independent of radius. The parameters determining the overall gravitational potential (essentially $v_{\phi c}$ ) can be determined from observations of stellar kinematics.

Estimates for the values of $\rho_{c}\left(r_{o}\right)$ (or $\left.\Sigma_{c}\left(r_{o}\right)\right)$ and $\Delta v_{c}$ can be derived from HI observations. 
Like the radial mass flux, the values of $\Delta v_{c}$ for the cold clouds and $\Delta v_{T}$ of the WHM turbulence will be determined by the SFR. Thus, we expect that for a universal (e.g., halo) gravitational potential, this family of model star-forming disks is primarily two-dimensional, with the two dominant parameters being the cool gas density at some point and the SFR.

The values of the mean WHM turbulence parameters $\Delta v_{T}$ and $\beta$ are the most difficult to evaluate. However, $\beta$ primarily enters the equations in the combination $(1+\beta) / \beta$, and so, whenever $\beta>$ afew, turbulent wave pressure dominates in the WHM and the exact value of $\beta$ does not greatly affect the other quantities. Moreover, $\beta$ is not independent of $\Delta v_{T}$ (both are functions of the SFR). The variable $\Delta v_{T}$, or a dimensionless combination like $\Delta v_{T} / v_{\phi c}$, can be viewed as a scaling parameter of the model, like the Mach number in shock hydrodynamics. Different mean WHM values, appropriate for galaxies with different SFRs, are obtained by changing the value of this parameter, as we will demonstrate.

Given our initial parameter values, we can now derive the scaling equations for the remaining variables. First, from the condition of vertical hydrostatic balance, we get the cool gas scale height,

$$
h_{c}=\left[\frac{\Delta v_{c}^{2}}{\pi G\left(\rho_{*}+\rho_{c}\right)}\right]^{1 / 2},
$$

where $\rho_{*}$ is the local star density. (The stars are assumed to have a larger scale height than the cool gas, so $\rho_{*}$ is essentially constant.)

Next, we use the continuity equation to derive $v_{r c}$, the cool gas inflow, and from equation (2) we derive the value of $v_{\phi c}$

$$
v_{\phi c}=\sqrt{\frac{G M_{o}}{r_{o}}} .
$$

This determines the last of the cool gas parameters, and we proceed to the remaining 
WHM quantities. Using equation (7) and the definition of $\beta$, we note that,

$$
\beta \mathbf{R} T_{w}=\Delta v_{T}^{2}
$$

This equation can be solved directly for $T_{w}$, but it also allows us to solve for the ratio of component scale heights. The scale height ratio equals the square root of the ratio of effective temperatures (see expression for $h_{c}$ above), which includes both a thermal and turbulent part in the case of the WHM. These parts are related by the previous equation, and so, we can write,

$$
\frac{h_{w}}{h_{c}}=\left(\frac{1+\beta}{\beta}\right)^{1 / 2} \frac{\Delta v_{T}}{\Delta v_{c}} .
$$

Using the temperature expression above in the pressure equation (eq. (7)), we get the related result for the component density ratio,

$$
\frac{\rho_{c}}{\rho_{w}}=\left(\frac{1+\beta}{\beta}\right) \frac{\Delta v_{T}^{2}}{\Delta v_{c}{ }^{2}} .
$$

The previous two equations can now be combined to give the component surface density ratio,

$$
\frac{v_{r w}}{-v_{r c}}=\frac{\Sigma_{c}}{\Sigma_{w}}=\left(\frac{1+\beta}{\beta}\right)^{1 / 2} \frac{\Delta v_{T}}{\Delta v_{c}}
$$

Finally, we return to the radial momentum equation (eq. 3) to determine $v_{\phi w}$. the left-hand side of this equation is zero, assuming constant mean radial velocities, and for the pressure gradient term the radial dependence of the pressure in this model $\left(P \propto \rho \propto r^{-3 / 2}\right)$ allows us to write, 


$$
\frac{r}{\rho_{w}} \frac{\partial P_{w}}{\partial r}=-\frac{3 P_{w}}{2 \rho_{w}}=-\frac{3}{2}\left(\frac{1+\beta}{\beta}\right) \Delta v_{T}^{2}
$$

Then substituting from equation (4) for the $\alpha$ term, multiplying by r, and dividing by $v_{\phi c}{ }^{2}$, the radial momentum equation can be written,

$$
\left(\frac{v_{\phi w}}{v_{\phi c}}\right)^{2}+\left(\frac{v_{r w}}{v_{\phi c}}\right)\left(\frac{v_{\phi w}}{v_{\phi c}}\right)-\left[1-\frac{3}{2}\left(\frac{1+\beta}{\beta}\right)\left(\frac{\Delta v_{T}^{2}}{v_{\phi c}^{2}}\right)\right]=0 .
$$

where we have also used the approximation that $G M_{o} /\left(r_{o} v_{\phi c}^{2}\right)=1$. This is a Bondi-Parker, accretion/wind equation, and is quadratic in the variable $\left(v_{\phi w} / v_{\phi c}\right)$. It is a central result of the model.

To evaluate that equation numerically, we use equation (13) for $v_{r w}$, and the continuity equation, with a given mass flux, for $v_{r c}$. As an example, let us assign the following representative values:

$M_{o}=2 \times 10^{11} M_{\odot}, \quad r_{o}=10 \mathrm{kpc} ., \quad \rho_{c}\left(r_{o}\right)=3.0 \mathrm{amu} / \mathrm{cm}^{3}, \Delta v_{c}=6.0 \mathrm{~km} / \mathrm{s}, \quad \Delta v_{T}=30 \mathrm{~km} / \mathrm{s}$, $\beta=3$, a mass flow of $\dot{M}=2.0 M_{\odot} y r .^{-1}$, and a stellar density of $\rho_{*}=\rho_{c}$

(used for computing scale heights).

Then we derive values of

$h_{c}=130 p c ., \quad h_{w}=770 p c ., \quad \rho_{w} / \rho_{c}=0.030, \quad v_{r c}=-3.4 \mathrm{~km} / \mathrm{s}, \quad v_{r w}=20 \mathrm{~km} / \mathrm{s}$, $v_{\phi_{c}}=290 \mathrm{~km} / \mathrm{s}, \quad v_{\phi_{w}}=277 \mathrm{~km} / \mathrm{s}$, and $T_{w}=21,000 \mathrm{~K}$.

Note that the radial velocities are very small compared to the azimuthal velocities, and also less than the turbulent velocity dispersions. Thus, we expect radial velocities to be quite difficult to observe.

If instead of the above value for $\Delta v_{T}$, we substitute a higher value of $\Delta v_{T}=50 \mathrm{~km} / \mathrm{s}$, 
we obtain values of $h_{w}=1300 p c ., v_{r w}=33 \mathrm{~km} / \mathrm{s}$, and $\Delta v_{\phi}=25 \mathrm{~km} / \mathrm{s}$. The thicker WHM layer and the larger azimuthal velocity difference in this case are both in accord with the observations of NGC 891. The surface density of the WHM relative to that of the cool gas increases linearly with $\Delta v_{T}$, consistent with the idea that the strong WHM emission in NGC 891 is the result of strong turbulence driven by the vigorous SF.

On the other hand, we noted above that Swaters et al. (1997) did not see such high velocity dispersions in their HI observations of NGC 891. However, these authors note that in the more nearly face-on galaxy NGC 6946, vertical velocities of up to $100 \mathrm{~km} / \mathrm{s}$ were detected by Kamphuis \& Sancisi (1993). These, and related observations, can be readily understood if the turbulent motions of HI gas in the thick disk and halo are primarily vertical. This situation is very natural if the most of the high dispersion HI gas is either entrained in local fountains and outflows, or is in the form of "high velocity clouds" consisting of cooled halo material falling back onto the disk (e.g., Benjamin 1999, Benjamin \& Danly 1997).

The impressive recent study of Thilker, Braun \& Walterbos (1998) on large HI shells in NGC 2403 also provides input on this question. These authors find mean in-plane shell expansion velocities of $26 \mathrm{~km} / \mathrm{s}$, and individual cases extending up to $56 \mathrm{~km} / \mathrm{s}$. We expect that much of the turbulent energy has already been vented in these large bubbles, and that the shells are observed in a deceleration stage.

Recent optical observations also provide evidence for the vigorous turbulence required by our model. Wang, Heckman \& Lehnert's (1997) spectroscopic study of the DIM in half a dozen nearby disks led them to suggest the existence of two components. The first is a 'quiescent DIM' with low ionization states and line widths of $20-50 \mathrm{~km} / \mathrm{s}$, versus the high ionization state, 'disturbed DIM' with line widths of $70-150 \mathrm{~km} / \mathrm{s}$. They suggest that the former is photoionized by diffuse $\mathrm{O}$ star radiation, while the latter is mechanically heated 
by supernovae and winds. In sum, turbulence with values of $\Delta v_{T} \simeq 50 \mathrm{~km} / \mathrm{s}$ or greater seems in accord with recent observations, and seems to yield very reasonable model values for actively star-forming disks.

\section{Star Formation Properties and Other Regularities in the Family of Models}

In constructing the model above, we have implicitly assumed that the SF law above threshold is constrained by a self-regulated heating and cooling balance. In the simplest case, we assume that all the important heating processes are directly proportional to the SFR (e.g., O star photoheating and turbulent wave heating). The important cooling

processes in the WHM include: 1) adiabatic cooling of high pressure gas elements, 2) radiative cooling in the mean WHM with a rate proportional to the WHM density squared, and 3) turbulent shock dissipation in the WHM, which depends on the SFR and gas density squared. The last two cooling rates generally scale with mean WHM gas density squared, and thus, require a similar density dependence in the SFR. That is, a Schmidt Law on average, albeit in density, rather than surface density. However, the processes involved are sufficiently complex that deviations from an $\mathrm{m}=2$ density power would not be surprising.

Thus, we write the following schematic equation for the heating and cooling balance for regions above the SF threshold,

$$
f_{S F} \rho_{c}^{2}=n_{w}^{2} \sigma c^{3} .
$$

The left-hand-side of this equation represents the Schmidt Law heating. The right-hand-side is a schematic collisional dissipation term, with "cross section" $\sigma$ and "sound speed" c. For example, this term could represent radiative cooling in the WHM via collisional excitation, with the temperature dependence contained within the factor $\sigma c^{3}$. However, if we make the 
approximation that $c \propto \Delta v_{T}$, and assume that $f_{S F}$ is universal, then the equation provides a scaling for the net dissipation cross section $\sigma$ in terms of $\Delta v_{T}$ and $\rho_{c} / \rho_{w}$. (See Appendix A for further discussion of the constrained SFR.)

There is a substantial literature on individual interstellar heating and cooling terms on many scales (e.g., see the recent discussions of Norman \& Ferrara 1996, and Ferriere 1998). Nonetheless, we still have a long way to go to fully understand the broad-band heating and cooling terms in the ISM. Any more specific formulation of the balance equation would probably require the introduction of insecure parametrizations with. It would also require additional physics, beyond that included in the simple model considered here. We will not pursue these topics in this paper, but merely point out that they may be easier to study in the context of the well-defined global structure provided by the model.

Equations (8)-(15) show that the model is a simple similarity solution to the hydrodynamic equations. In the limit of small radial velocities and small turbulence (and thus little WHM component) the model must be essentially the same as the self-similar, viscous, Mestel disks studied recently by Bertin (1997) and Mineshige \& Umemura (1996). (Our model also has some similarities to the one-component convective model of Waxman (1978).) The self-similar structure of the model helps to understand the universal properties of gas-rich galaxy disks, like the Tully-Fisher relation between maximum circular velocities and the total luminosities of disk galaxies (e.g., Courteau 1997 and references therein), and universal gas density profiles (see e.g., the extensive HI study of Broeils \& Rhee 1997).

Eisenstein and Loeb (1996) point out that the small dispersion in the observational Tully-Fisher relation suggests the operation of a "strong feedback process" that "regularize(s) SF and gas dynamics," like the model presented here. On the other hand, Mo et al. (1998) believe that the small Tully-Fisher scatter could in fact come out of early galaxy formation processes. Even so, Eisenstein and Loeb are probably also correct if a 
large fraction of galaxies experience merger events subsequent to their formation. That is, while we expect galaxy collisions and mergers to disrupt the "universal" disk structure, turbulent self-regulation will re-establish it.

Some of these questions are answered by the new numerical hydrodynamical models of galaxy formation in several cosmologies by Elizondo et al. 1999). These models included multiple gas phases and supernova feedback. The authors found that the Tully-Fisher scatter of their model galaxies was within the acceptable both with and without the feedback effects included. However, only the feedback models reproduced the correct slope of the Tully-Fisher relation, and the slope was quite sensitive to the feedback amplitude.

Another regularity, Freeman's Law, states that high surface brightness galaxies all have about the same central surface brightness, or that disk galaxies have a maximum central surface brightness (see Courteau 1996 and references therein). This too would seem to be a natural consequence of large-scale SF regulation, albeit in the central regions of the disk. In a number of nearby starburst galaxies the gas surface density continues to follow a power-law as far into the center as it can be resolved (Struck-Marcell 1991, Young et al. 1995). In many cases this may be the result of gas inflow driven by a bar component or other disturbance. In many other late-type disks the gradient in the surface density flattens to a value of about 10 solar masses per square parsec in the central, rising rotation curve region (though often with a central, molecular gas spike, see Young et al. 1995). We speculate that this latter case represents a normal quiescent state. Disks of both profile types are observed to have a comparable value of $\Sigma_{c}$ at the radius where the rotation curve flattens (Broeils \& Rhee 1997). We conjecture that Freeman's Law may be the result of the fact that most stars form in the centers of bright, late-type disks at a rate appropriate to this gas density, and over comparable timescales. 


\section{Conclusions}

The following list summarizes the properties and some probable consequences of the models presented in this paper.

1) A $1 / r$ surface density profile is assumed in both thermal phases (on the basis of the Least Dissipation Principle and other arguments, see section 2.2). It is also assumed that vertical scale heights are determined by local self-gravity, and so, increase slowly with radius $\left(\right.$ as $\propto r^{1 / 2}$ ). This implies that mean volume densities scale as, $\rho_{i} \propto r^{-3 / 2}$.

2) Thus, the ratio of gas phase densities are constant across the star-forming region of the galaxy disk. The model predicts that the value of this ratio depends on the amount of turbulence, and specifically, on the parameter $\Delta v_{T} / \Delta v_{c}$.

3) We assume that the circular velocities of each gas phase are constants independent of radius. The equations of state and the assumption that all momentum equation terms have the same radial scaling implies that the remaining velocities, $v_{r c}, v_{r w}, \Delta v_{T}, \Delta v_{c}$, are also constant with radius.

4) In general, the model allows all of the velocities in each phase - azimuthal, radial, and dispersive - to have different (non-zero) values. The NGC 891 effect of different rotational velocities as a function of height above the disk (Swaters et al. 1997) is predicted to be generic in turbulent disks.

5) The model predicts a hierarchy in velocity magnitudes in each phase, i.e., azimuthal velocities $>>$ velocity dispersions $>$ radial velocities. The low value of the latter will make them difficult to observe. This hierarchy is the result of the similarity equations and the simple wind/accretion equation (eqs. (8) - (15)).

6) However, even such low velocity radial flows are consistent with mass fluxes comparable to typical SFRs in late-type disks. In the absence of radial flows, we would 
expect gas consumption at smaller disk radii, to modify the gas density profile. Radial replenishment can prevent this and effectively distribute the consumption across the disk. The radial flow may also draw on reservoirs of gas in the non-star-forming outer disk, further increasing the global consumption time. Evolutionary effects will be considered in a later paper.

7) Similarly, galactic abundance gradients will be smoothed by the large-scale radial flows. The simple closed-box model of chemical evolution within isolated disk annuli is not appropriate in the context of these radial circulation models. However, the quantitative effects of radial flow are complicated by the fact that the flows are slow. E.g., with a radial flow velocity of order $3 \mathrm{~km} / \mathrm{s}$, the timescale for a gas element to cross a disk of radius $10 \mathrm{kpc}$ is a few billion years. Moreover, motion of a gas element will generally be partly advective and partly diffusive in this turbulent environment. Thus, the typical smoothing time may be only a little less than the typical disk age.

8) The model requires a balance between heating and cooling. Heating is primarily the result of SF activity, and most cooling terms depend on the second power of the gas density (assuming the constant phase balances of the model). Thus, a Schmidt Law SFR is the natural result of the thermohydrodynamical balance.

9) If the Schmidt Law (or a related parametrization, such as that of Silk and Wyse, see Silk 1997) is in fact a consequence of global hydrodynamic self-regulation, then there are some immediate corollaries. Perhaps, the most important is that transient, burst modes of SF are possible when disturbances take galaxies far from the regulated state. Thus, SF phenomenologies may be very different in highly disturbed galaxies (e.g., Struck-Marcell \& Scalo 1987), or during galaxy formation. On the other hand, the Schmidt parametrization may be marginally valid in environments where, $r / \Delta v_{T}=\tau_{\text {relax }}<\tau$, with $\tau$ defined as an appropriate "age". For example, the Schmidt Law may work in waves in both grand design 
and collisional galaxies if the wave crossing time is longer than $\tau_{\text {relax }}$. The same argument may be valid in the centers of major merger remnants.

10) Global regularities in star-forming disks, such as the Tully-Fisher relations and the Freeman Law, may also be the result of global self-regulation, of the type inherent in the present models. They may also be the result of formation processes, including turbulent self-regulation during formation. Continuing self-regulation is important for maintaining the global regularities, and restoring them following a disturbance.

11) The model equations can readily be generalized beyond the two-phase version described here to include a continuous range of phases. More sophisticated treatments of viscosity, turbulence, heating and cooling processes can be included, much as detailed nuclear rates and opacities are included in stellar evolution models.

In sum, the hydrodynamic similarity model presented above is an attempt to bring together the essential thermohydrodynamical processes needed for a coherent conceptual picture of actively star-forming galaxy disks as self-regulated, multiphase, "dissipative structures." The basic hydrodynamic structure of each phase is much like that of an isothermal polytrope, but these are not quasi-static, equilibrium states. The model assumes that there are turbulent flows on many scales, and the WHM is more accurately viewed as a set of locally adiabatic states, with specific entropy gradients. The disk structure described by this model, with gas profiles regulated in accord with minimal dissipation and transport, and SF simultaneously sustained and moderated by slow, radial flows, probably describes most late-type disk galaxies.

Acknowledgements: We are very grateful to Julia K. Burzon for creating Figure 1. We also want to thank P. N. Appleton, R. Benjamin, V. Charmandaris, B. Elmegreen, L. Sage, J. Scalo, J. M. van der Hulst and especially an anonymous referee and our editor S. Shore for providing helpful (regulating!) feedback. We are also grateful for partial page charge 
support from an American Astronomical Society Small Grant.

Appendix A: A Plausibility Argument for Radial Entropy Increase and the Schmidt Law

The positive radial entropy gradients in the WHM component of the model presented above may seem unphysical, especially since this gas is expanding in an average outward flow. However, there is an obvious entropy source in the nonlinear acoustic waves that partially support this flow. At the same time, radiative cooling provides an obvious entropy sink, yet when this gas experiences significant cooling it is generally transformed into the cool component. Thus, on average, the entropy of gas that stays in the WHM either increases, or is balanced by adiabatic expansion.

Specifically, consider a non-cooling element of the WHM moving outward in the mean flow above the midplane of the disk. Suppose for simplicity, that its specific entropy is significantly increased only when it passes directly over a young star cluster, assuming such star clusters are the primary source of shock turbulence. Then, the rate at which the entropy of that element is increased will be proportional to the number of young clusters it passes over per unit time. (Note that because of the reduced azimuthal velocity of the WHM, the element will pursue a spiral trajectory as viewed in a reference frame comoving with the midplane gas.)

If the SFR is described by a Schmidt Law, and assuming most new stars are born in clusters, then the number of clusters within a thin annulus of width $\Delta r$, at radius $\mathrm{r}$ is,

$$
N_{c l}=2 \pi r \Delta r\left(\frac{\psi}{M_{c l}}\right) \tau_{c l}=c_{1} \frac{\Delta r}{r^{1+m}}
$$

In this equation, $\psi$ is the usual SFR (mass of stars produced per unit area per unit time), $M_{c l}$ is the mean cluster mass, and $\tau_{c l}$ is the mean lifetime of the massive stars in the 
cluster. The final equality assumes a Schmidt Law of the form, $\psi \propto \Sigma_{c}^{2+m}$, and a surface density profile of the form $\Sigma_{c} \propto 1 / r$. The constant $c_{1}$ is the combination of all constants in the previous equality.

We further assume that the gas element expands in the azimuthal direction by an amount proportional to $\mathrm{r}$ as it moves outward. This is just the expansion that is required to maintain the assumed surface density profile. It also guarantees that the WHM element covers a constant fraction of each thin annulus it crosses. Thus, the gas element crosses a constant fraction of the clusters $N_{c l}$ in each thin annulus, and it is reasonable to assume that the rate of shock hits and entropy increase it experiences is proportional to this annular cluster fraction. Thus, the net entropy increase in traveling from radius $r_{1}$ to radius $\mathrm{r}$ is

$$
\Delta S \simeq c_{2} \int \frac{d r}{r} \simeq c_{2} \log \left(r / r_{1}\right)
$$

in the case $\mathrm{m}=0$. The constant $c_{2}$ contains the product of the earlier constant $c_{1}$, the annulus fraction covered by the gas element, and the mean entropy input per cluster.

For a perfect gas,

$$
S=\log \left(P / \rho^{\gamma}\right)=\log \left(K_{V}(r)\right)
$$

where the second equality makes use of equation (6), in section 2.3, (and the subscript V emphasizes that these are volume quantities). Assuming that this equals the preceding equation to within an additive constant, we have,

$$
K_{V}(r) \propto r^{c_{2}}
$$

As noted in section 2.3 the quasi-steady model presented above requires that $c_{2}=1$. One factor contained within the constant $c_{2}$ is the magnitude of the SFR, e.g., the SFR at 
a particular radius. Thus, the self-regulating feedback processes can adjust the SFR in such a way that $c_{2}$ is driven towards unity, giving the desired entropy gradient.

Therefore, the fact that the entropy gradient is a power-law follows from the $m=0$ spatial dependence of the Schmidt Law SFR, while the value of the power depends on the magnitude of the SFR. In other words, the entropy gradient required for a hydrodynamic steady state can be achieved by feedback adjustments to the amplitude and spatial dependence of the SFR.

Now let us consider the effects of these self-regulating processes from a slightly different point of view. According to equations (6) and (7) the mean WHM temperature scales as, $T_{w} \propto(1+\beta) K_{V} \rho^{2 / 3}$. For a globally adiabatic gas, $\mathrm{K}$ is constant, and $T_{w} \propto 1 / r$ (for constant $\beta$ ). While, as described above, for a locally adiabatic gas, with a radial entropy gradient such that $K_{V} \propto r$, we have $T_{w}$ constant. If the latter alternative does not obtain, then the variation of scale height with radius will be different than the $h \propto r^{1 / 2}$ form assumed above. Qualitatively, if the scale height increases less rapidly, then warm gas remains closer to the midplane and is denser, so cooling rates are increased. If more of this gas goes into the cool phase, we expect that the SFR will increase (relative to the quasi-steady model), driving increased turbulence and heating, and increasing the scale height.

Conversely, if the scale height increases more rapidly with radius, the SFR will be less than in the steady model, eventually diminishing pressure support, and reducing the scale height. More generally, because of the temperature dependence of the cooling rates, significant temperature gradients would likely result in pressure imbalances, which would lead to time-dependent convection (as well as thermal conduction). That is, temperature gradient states do not generally satisfy the steady state equations above.

These considerations are qualitative, and have loopholes, but they do show why global states with modest entropy gradients would be preferred. They also provide some insight 
into how closely the Schmidt Law SFR is connected to such states. 


\section{REFERENCES}

Benjamin, R. A. 1999, in Stromlo Workshop on High-Velocity Clouds, ASP Conference Series 166, eds. B. K. Gibson \& M. E. Putnam (ASP: San Francisco), p. 147

Benjamin, R. A. \& Danly, L. 1997, ApJ, 481, 764

Bertin, G. 1997, ApJ, 478, L71

Bregman, J. N. 1980, ApJ, 236, 577

Brinks, E. 1990, in The Interstellar Medium in Galaxies, eds. H. A. Thronson, Jr. \& J. M. Shull (Dordrecht: Kluwer), p. 39

Broeils, A. H., \& Rhee, M.-H. 1997, A\&A, 324, 877

Bertoldi, F., \& McKee, C. F. 1997, Rev. Mex. AAp Serie de Conferencias, Vol. 6, 1st Guillermo Haro Conference on Astrophysics: Starburst Activity in Galaxies, p. 195

Chappell, D. W., \& Scalo, J. M. 1997, ApJ, submitted (preprint: astroph 9707102)

Courteau, S. 1996, ApJS, 103, 363

Courteau, S. 1997, AJ, 114, 2402

Cox, D. P. 1981, ApJ, 245, 534

Dalcanton, J. J., Spergel, D. N., \& Summers, F. J. 1997, ApJ, 482, 659

Diaz-Miller, R. I., Franco, J., \& Shore, S. N. 1998, ApJ, 501, 192

Dickey, J. M. \& Lockman, F. J. 1990, ARA\&A, 28, 215

Dopita, M. A. 1985, ApJ, 295, L5

Dopita, M. A. 1990, in The Interstellar Medium in Galaxies, eds. H. A. Thronson, Jr. \& J. M. Shull (Dordrecht: Kluwer), p. 437

Dopita, M. A., \& Ryder, S. D. 1994, ApJ, 430, 163 
Eisenstein, D. J., \& Loeb, A. 1996, ApJ, 459, 432

Elizondo, D., Yepes, G., Kates, R., Muller, V., \& Klypin, A. 1999, ApJ, 515, 525

Elmegreen, B. G. 1997, ApJ, 477, 196

Ferguson, A. M. N., Wyse, R. F. G., \& Gallagher, J. S., Jr. 1996, AJ, 112, 2567

Ferriere, K. 1998, ApJ, 497, 759.

Franco, J., \& Shore, S. N. 1984, ApJ, 285, 813

Haynes, M. P. \& Broeils, A. H. 1997, in The Interstellar Medium in Galaxies, ed. J. M. van der Hulst (Dordrecht: Kluwer), p. 75

Heiles, C. 1984, ApJS, 55, 585

Hoopes, C. G., Walterbos, R. A. M., Greenwalt, B. E. 1996, AJ, 112, 1429

Howk, J. C., \& Savage, B. D. 1997, AJ, 114, 2463

Hunter, D. A., \& Gallagher, J. S. III 1997, ApJ, 475, 65

Kamphuis, J. J., \& Sancisi, R. 1993, å, 273, L31

Kennicutt, R. C., Jr. 1989, ApJ, 344, 685

Kennicutt, R. C., Jr. 1990, in The Interstellar Medium in Galaxies, eds. H. A. Thronson, Jr. \& J. M. Shull (Dordrecht: Kluwer), p. 405

Kennicutt, R. C., Jr. 1998a, ApJ, 498, 541

Kennicutt, R. C., Jr. 1998b, in Galaxies: Interactions and Induced Star Formation, Saas Fee Advanced Course 26, eds. D. Friedli, D. Martinet, \& D. Pfenniger (Berlin: Springer), p. 1

Larson, R. B. 1979, MNRAS, 186, 479

Lehnert, M. D., \& Heckman, T. M. 1996, ApJ, 462, 651

Li, F., \& Ikeuchi, S. 1992 ApJ, 390, 405 
Mac Low, M., McCray, R., \& Norman, M. L. 1989, ApJ, 337, 141

Malhotra, S. 1995, ApJ, 448, 138

Martin, C. L. 1998, ApJ, 506, 222

McKee, C. F. 1993, in Back to the Galaxy, eds. S. S. Holt \& F. Verter (New York: A. I. P.), p. 499

McKee, C. F., \& Ostriker, J. P. 1977, ApJ, 218, 148

Mineshige, S., \& Umemura, M. 1996, ApJ, 469, L49

Minter, A. H., \& Spangler, S. R. 1996, ApJ, 458, 194

Minter, A. H., \& Balser, D. S. 1997, ApJ, 484, L133

Minter, A. H., \& Spangler, S. R. 1997, ApJ, 485, 182

Mo, H. J., Mao, S. \& White, S. D. M. 1998, MNRAS, 295, 319

Noguchi, M. 1998, Nature, 392, 253

Norman, C. A., \& Ferrara, A. 1996, ApJ, 467, 280

Norman, C. A., \& Ikeuchi, S. 1989, ApJ, 345, 372

Parravano, A. 1988, A\&A, 205, 71

Parravano, A. 1989, ApJ, 347, 812

Passot, T., Vázquez-Semadeni, E., \& Pouquet, A. 1995, ApJ, 455, 536

Rand, R. J. 1996, ApJ, 462, 712

Rand, R. J. 1997a, in The Interstellar Medium in Galaxies, ed. J. M. van der Hulst (Dordrecht: Kluwer), p. 105

Rand, R. J. 1997b, ApJ, 474, 129

Rand, R. J. 1998, ApJ, 501, 137 
Reynolds, R. J. 1996, in The Physics of Galactic Halos, eds. H. Lesch, R.-J. Dettmar, U. Mebold, \& R. Schlickeiser (Berlin: Akademie Verlag), p. 57

Rosen, A., \& Bregman, J. N. 1995, ApJ, 440, 634

Samland, M., Hensler, G., \& Theis, Ch. 1997, ApJ, 476, 544

Scalo, J. M. 1987, in Interstellar Processes: Proceedings of the Symposium, eds. D. J. Hollenbach \& H. A. Thronson, Jr. (Dordrecht: Reidel), p. 349

Scalo, J. M., \& Struck-Marcell, C. 1984, ApJ, 276, 60.

Schulman, E., Bregman, J. N., \& Roberts, M. S. 1994, ApJ, 423, 180

Shapiro, P. R., \& Benjamin, R. A. 1991, PASP, 103, 923

Shapiro, P. R., \& Field, G. B. 1976, ApJ, 205, 762

Silk, J. 1997, ApJ, 481, 703

Steinmetz, M., \& Müller, E. 1995, MNRAS, 276, 549

Struck-Marcell, C. 1991, ApJ, 368, 348

Struck-Marcell, C., \& Scalo, J. M. 1987, ApJS, 64, 39

Swaters, R. A., Sancisi, R., \& van der Hulst, J. M. 1997, ApJ, 491, 140

Tenorio-Tagle, G., Rozyczka, M., \& Bodenheimer, P. 1990, A\&A, 237, 207

Thilker, D. A., Braun, R., \& Walterbos, R. A. M. 1998, å, 332, 429.

Tomisaka, K. 1992, PASJ, 44, 177

van der Hulst, J. M. 1996, in The Minnesota Lectures on Extragalactic Neutral Hydrogen, A.S.P. Conf. Series 106, ed. E. D. Skillman (San Francisco: A. S. P.), p. 47 van der Hulst, J. M. (ed.) 1997, The Interstellar Medium in Galaxies (Dordrecht: Kluwer) Wang, J., Heckman, T. M., \& Lehnert, M. D. 1997, ApJ, 491, 114 
Waxman, A. M. 1978, ApJ, 222, 61

Young, J., et al. 1995, ApJS, 98, 219

\section{FIGURE CAPTIONS}

Fig. 1. - Schematic illustrating local fountain flows, cooling filaments, and the global radial flows in a model disk (courtesy Julia K. Burzon) 
This figure "f1.gif" is available in "gif" format from: http://arxiv.org/ps/astro-ph/9907425v1 